\title{
D eveloping products for personalized medicine: NIH Research Tools Policy applications
}

\section{Ranjan Gupta, JP Kim, Jack Spiegel \& Steven M Ferguson ${ }^{\dagger}$}

${ }^{\dagger}$ Author for correspondence O ffice of Technol ogy Transfer, $\mathrm{N}$ ational Institutes of $\mathrm{H}$ ealth, $D$ epartment of $\mathrm{H}$ ealth and H uman Services, 6011 Executive Boulevard, Suite 325, Rockville, MD 20852, USA Tel: +1 301435 5561; Fax: +1 301402 0220; E-mail: sfoh@nih.gov

Keywords: Bayh-D ole Act, $\mathrm{NIH}$, personalized medicine, research tools

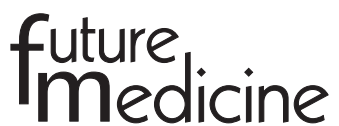

For innovators at academic or industrial institutions to develop personal ized medicine products at the accelerated pace required, rapid access to state-of-the-art research tools and reagents are needed. Unreasonable restrictions or delays in the use of such tools may undermine the development of these customized diagnostics and therapeutic products designed to provide significant treatment benefits to patients who frequently do not benefit from traditional therapeutic approaches. In its funding agreements and its own internal research programs, the National Institutes of Health is implementing policies to facilitate the exchanges of these research tools and related resources.

\section{Background}

As the world's population has increased over time, medicine has tried to achieve a practical approach of doing the most good for the most people. To meet the needs of patient populations requiring medical attention and to provide incentives for organizations to research and develop new and effective medical treatments and other regimens, approaches were standardized to meet largely single economies of scale and to accommodate the largest populations possible within the same generic therapeutic regimen range, much like $\mathrm{H}$ enry Ford and the manufacturing of the Model $T$ automobile. Unfortunately, those patients with responses that lie at the extreme ends of the bell curve are not well served by this model. Certain patients remain less than optimally treated while others may suffer adverse reactions, including possibly death.

0 ver the years, it has been recognized that there is variability in human responses and that not all therapeutics will be equally effective and beneficial for all patients. Yet, human variability of responses has remained a 'black box', which has not always been effectively and economically discernible. As time has progressed, technologies have devel oped and new research resources have become available for the identification of such human variability, as well as for the development of specific treatments directed to the individual - 'personalized medicine'. To achieve the necessary customization of diagnostic tests and therapeutics for such small target populations, the biomedical enterprise will require new research and development $(R \& D)$ efforts focused in the areas of gene profiling and association studies between drug response and sequence variation. This multiple, but often parallel, research can only be effective with rapid access to the necessary research tools in order for innovation to provide the most personalized healthcare.

Personalized medicine and implications for drug development

It is commonly known that while most people have no adverse reactions with the variety of foods and beverages consumed everyday, some individuals within the population may show severe allergic reactions, such as to shellfish or peanuts. For example, it is known that many East Asians lack the aldehyde dehydrogenase-2 (ALDH2) enzyme and thus may experience unpleasantness when consuming alcohol as acetaldehyde accumulates in the system [1]. Yet, most Westerners (C aucasians) can consume alcohol without such effects because this enzyme is available in their system. In much the same way, people in the field of medicine have known for a long time that while a given drug may have a remarkable effect on many (drug responders), it may have little or no effect on others (nonresponders). The same drug may even have major adverse effects on other individuals.

Starting with Archibald Garrod's hypothesis in 1902 [2], linking a genetic defect to an error in metabolism (via enzymatic defect) in the case of alcaptonuria, and Snyder's report in 1932 [3] on 'taste blindness' to phenylthiocarbamide (PTC), which linked genetic polymorphism to variability in human sensory perceptions, the history of formalized pharmacogenetics (a term coined by Friedrich Vogel in 1959 [4]) and, subsequently, pharmacogenomics is a fascinating tale of discoveries [5-7]. Volumes of research literature now exist demonstrating the role of genetic differences in human drug response. 
Such differences may account for the variability in drug absorption (e.g., affinity and binding to cell receptors, drug uptake, and drug transport), drug metabolism, and drug excretion. All these physiological processes are governed by a number of genes, each susceptible to mutation. $G$ enetic defects leading to deficiencies in drug-metabolizing enzymes, such as cytochrome P450 (CYP) gene products, are often implicated in adverse drug reactions (ADRs). Pharmacogenetics has focused its approach on candidate genes and gene products whose defects may lead to altered drug response; hence, this approach is monogenic.

$W$ ith the complete sequencing of the human genome, as a result of the $\mathrm{H}$ uman $\mathrm{G}$ enome Project (H GP), scientists are now able to look at a bigger picture, focusing not just on single genes but on the function of the genome overall. This has, thus, given birth to 'pharmacogenomics' (and toxicogenomics) - the study of drug response and toxicity in relation to the sequence variation of all genes in the human cell (i.e., the genome). The study of human genomic variation (polymorphisms) has significantly enhanced our understanding of human biology. Variations in the human genome correlate, for instance, with ethnicity/race and the geographic distribution of populations, all of which have major implications for drug discovery and development. It is becoming increasingly clear that the 'one-drug-fits-all' approach is no longer suitable. H ence, the 21st century may well be the age of personalized medicine the practice of drug development and administration based on our understanding of individual differences in patients through the knowledge of pharmacogenetics and pharmacogenomics [8]. In fact, pharmacogenomics expands upon pharmacogenetics and the terms are often used synonymously. Therefore, for the remaining article, we will simply use the term pharmacogenomics in our further discussions on personalized medicine.

It is estimated that ADR is the fourth leading cause of death in the USA [9]. Given the immense cost of drug discovery, both in time and money, major pharmaceutical corporations depend on a handful of blockbuster drugs that can serve a large target patient population and raise billions of dollars in revenue that may help recover the investment made not only in that particular success but also in those that failed. Yet, a single fatality due to ADR may be sufficient to jeopardize this investment, as well as the benefit to actual responders to treatment. To avoid such circumstances, it might beadvantageous for a drug company to take an individualized approach.

If genetic tests, based on pharmacogenomics, are available to screen patients prior to drug administration so as to determine whether the given drug may or may not be suitable for a given patient, this would reduce the incidences of AD R and save the drug developer from litigation, bad publicity and even the risk of having to withdraw a drug from the market. In fact, it may even help to increase the market size for some drugs [10]. As noted earlier, customization of diagnostic tests and therapeutics for small target populations will require large-scale $R \& D$ in areas of gene profiling, as well as in association studies between drug response and sequence variation. For innovations to move at the fast pace required, developers of customized diagnostic tests and therapeutics will need to have rapid access to current research tools and reagents.

\section{Research tool applicationsfor personalized medicine}

To examine this last issue we shall now focus our attention on the various $R \& D$ procedures currently being used in pharmacogenomics and their upcoming trends. The study of the human genome and its variation over the last two decades has proceeded simultaneously with the innovation of a variety of laboratory techniques and instrumentation. The advent of the automated DNA sequencing and PCR using automated thermal cyclers has expedited the sequencing of the genome by orders of magnitude. For studying sequence variations in specific regions of the genome, techniques have evolved to analyze, for example, microsatellite DNA and single-stranded conformational polymorphisms (SSCPS). Use of expressed sequence tags (ESTs), cDN As, antisense molecules, small interfering RNAs (siRN As), full-length genes and their expression products and haplotypes have added new dimensions to genomic research. The most recent in this sequel is the study of SN Ps - a most abundant form of variation in the human genome, occurring at a fre quency of $1 \%$ or more, which distinguishes them from rare point mutations. SN Ps account for over $90 \%$ of the differences between individuals, and large-scale association studies between SN Ps and drug responses have become a major approach to pharmacogenomic studies. An extension of the SN P map is a haplotype map consisting of blocks of tightly linked SN PS 
charted along the human genome. Genotyping SN Ps on a large-scale to obtain statistically meaningful associations requires high-throughput techniques that can screen hundreds of thousands of SN Ps for thousands of individuals over a short period of time. For this, conventional gel methods have been replaced by automated oligonucleotide chip technology that provides accurate and specific information on a microarray. M icroarray technology is also being exploited to study gene-expression profiling that provides high-through put information regarding the status of expression of thousands of genes within a given cell at a given time. Such analyses are also highly meaningful in understanding phenotypic associations to, for example, disease conditions and drug response. Advances in these techniques have relied upon advances in nucleic acid isolation, amplification, hybridization and detection technologies and fluorescent probe technologies, as well as on the development of highly sophisticated computer software in bioinformatics for the analysis of the volumes of genetic data generated by these procedures.

It is evident that this science of the future will require greater interdependence between basic and applied research, between academia and industry, and greater interdisciplinary cooperation through sharing of data, expertise, resources, and tools. An effective public policy for the exchange and sharing of such resources is, thus, a necessary element for the effective development of diagnostics and therapeutics for personalized medicine.

Intellectual property, research tools, and personalized medicine products

An invention may be a marketable product for one enterprise, while being an essential research tool for another to proceed to the next phase of innovation. Tangible property in the form of products, such as a model organism or a research reagent kit, may be closely tied to the intellectual property (IP). H owever, while the ownership of the tangible property may lie with whoever developed or purchased it, the IP may be owned by a separate entity. Since IP rights invariably dominate the ownership of the tangible property, the IP rights need to be exercised appropriately, such as through judicious licensing practices, so as not to encumber the free sharing of tools for non-profit research. IP rights need to be administered properly to avoid inappropriate commercialization option rights, reach-through royalty or product reach-through rights that may impede or discourage further research. There fore, the appropriate management of IP becomes a critical rate-limiting factor in the path of drug discovery that could make or break our endeavors toward personalizing medicine.

To understand these IP issues so that they may be judiciously applied in developing products for personalized medicine, we provide here the perspectives from the $\mathrm{N}$ ational Institutes of $\mathrm{H}$ ealth (NIH) on what constitutes 'research tools' and how they may be shared for seamless growth in $R \& D$ without compromising proprietary rights where they may duly need to be exercised. The following discussion outlines the strategy imple mented in NIH federally funded research and showcases examples of some $\mathrm{NIH}$ practices for inventions with potential personalized medicine applications. H owever, others may find alternative approaches to achieve similar desirable end points or consider the $\mathrm{NIH}$ practices as suited to their individual circumstances.

$\mathrm{NIH}$ research tool policy implicationsfor personalized medicine

The NIH publication 'Sharing Biomedical Research Resources: Principles and Guidelines for Recipients of NIH Research Grants and Contracts', also referred to as the 'N IH Research Tools Policy' [11], defines research tools very broadly, recognizing that the same resource may serve as a research tool in one case and a product in another. Such tools may include cell lines, model organisms, monoclonal antibodies, reagents, growth factors, combinatorial chemistry and D N A libraries, clones and cloning tools (e.g., PCR), experimental methods, laboratory equipment and machines, computer software, and databases. All of these have important uses in the $R \& D$ of personalized medicine products. Generally, researchers can evaluate if a given resource is a research tool by determining whether:

- the primary usefulness of the resource is as a tool for discovery rather than a product approved by the US Food \& D rug Administration (FD A) or an integral component thereof

- the research is a broad enabling invention that will be useful to many scientists (or multiple companies in developing multiple products) rather than a project-specific or productspecific resource

- most importantly, whether the resource is readily usable and distributable as a tool without the need for private-sector involvement for further development and distribution 
Box 1. Four key principles of NIH research tool policy applicable to R\&D for personalized medicine.

- Ensure academic freedom and publication.

- Ensure appropriate implementation of the Bayh-Dole Act.

- M inimize administrative impediments to research.

- Ensure dissemination of research resources developed with NIH funding.

Research tools for personalized medicine generally have a short life cycle for usability and may be patented or non-patented. Some additional perspectives are also discussed in the N IH publication 'D eveloping Sponsored Research Agreements: Considerations for Recipients of N IH Grants and Contracts' [12]. It should be noted that certain resources might have dual uses. For example, potential therapeutic products may also have secondary uses, such as research tools; hence, each use needs to be evaluated. To identify an appropriate strategy for making such resources available as 'research tools' in personalized medicine research, scientists should consider whether further research, development and private investment are needed to realize this usefulness.

As part of the US Public $\mathrm{H}$ ealth Service (PH S), the N IH strives to achieve a fine balance between maintaining scientific freedom in $R \& D$ and fulfilling the requirement of the Bayh-D ole Act (1980) [13] for the interest of improving public health and promoting innovation without unduly encumbering future research and discovery. The NIH recognizes that the transfer of technologies with applications in personalized medicine and other areas of research must balance the rewards of broad IP protection afforded to founders of enabling genomic inventions with the benefits of fostering opportunities to those striving to improve upon those innovations. Thus, research for new diagnostic and therapeutic products for personalized medicine, similar to general drug discovery efforts [14], can readily use the following NIH Research Tools Policy Principles (Box 1).

Principle 1: Ensure academic freedom and publication

Academic research freedom based upon collaboration and the scrutiny of research findings within the scientific community are at the heart of the scientific enterprise. Institutions that receive $\mathrm{NIH}$ research funding through grants, cooperative agreements or contracts (recipients) have an obligation to preserve research freedom, safeguard appropriate authorship, and ensure timely disclosure of their scientists' research findings through, for example, publications and presentations at scientific meetings. Recipients of N IH funding are expected to avoid signing agreements that unduly limit the freedom of investigators to collaborate and publish, or that automatically grant co-authorship or copyright to the provider of a material.

Reasonable restrictions on collaboration by academic researchers involved in sponsored research agreements with an industrial partner that avoid conflicting obligations to other industrial partners are understood and accepted. Similarly, brief delays in publication may be appropriate to permit the filing of patent applications and to ensure that confidential information obtained from a sponsor or the provider of a research tool is not inadvertently disclosed. H owever, excessi ve publication delays or requirements for editorial control, approval of publications or withholding of data all undermine the credibility of research results and are unacceptable.

\section{Principle 2: Ensure appropriate} implementation of the Bayh-Dole Act When the NIH funds an institution's research work, the activity is subject to various laws and regulations, including the Bayh-D ole Act [14]. Generally, funding recipients are expected to maximize the use of their research findings by making them available to the research community and the public, and through their timely transfer to industry for commercialization through licensing or other means.

The right of funding recipients to retain a title to inventions made with $\mathrm{N} \mathrm{IH}$ funds comes with the corresponding obligations to promote the utilization, commercialization and public availability of these inventions. The Bayh-Dole Act encourages funding recipients to patent and license subject inventions as one means of fulfilling these obligations. H owever, the use of patents and exclusive licenses is not the only nor, in some cases, the most appropriate means of implementing the Act. Where the subject invention is useful primarily as a research tool, inappropriate patent and licensing practices are likely to thwart rather than promote utilization, commercialization and public availability of the invention.

In determining an IP strategy for a $\mathrm{N} \mathrm{IH} \mathrm{-funded} \mathrm{invention} \mathrm{that} \mathrm{is} \mathrm{useful} \mathrm{primarily} \mathrm{as}$ 
a research tool, organizations should analyze whether further research, development and private investment are needed to real ize this primary usefulness. If it is not, the goals of the Bayh-D ole Act can be met through publication, a deposit in an appropriate databank or repository, wide spread non-exclusive licensing, or any other number of dissemination techniques. Restrictive IP rights or licensing of such an invention, such as to a for-profit sponsor for exclusive internal use, is antithetical to the goals of the Bayh-D ole Act. Where private sector involvement is desirable to assist with maintenance, reproduction and/or distribution of the tool, or because further research and development are needed to realize the invention's usefulness as a research tool, licenses should be crafted to fit the circumstances, with the goal of ensuring widespread and appropriate distribution of the final tool product. Exclusive licensing of such an invention, such as to a distributor that will sell the tool or to a company that will invest in the development of a tool from the nascent invention, can be consistent with the goals of the Bayh-D ole Act.

Principle 3: Minimize administrative impediments to academic research

Each iteration in a negotiation over the terms of a license agreement or materials transfer agreement delays the moment when a research tool may be put to use in the laboratory. Funding recipients should take every reasonable step to streamline the process of transferring their own research tools freely to other academic research institutions using either no formal agreement, a cover letter, the Simple Letter Agreement of the Uniform Biological Materials Transfer Agree ment (UBM TA), or the UBM TA itself [101]. The $\mathrm{NIH}$ has published an updated free-standing version of the Simple Letter Agreement [102] and strongly encourages its use for transfers of unpatented research materials.

Where they have not al ready done so, institutions receiving $\mathrm{N} \mathrm{IH}$ funds should develop and implement clear policies that articulate acceptable conditions for acquiring research-tool resources and refuse to yield on unacceptable conditions. The N IH acknowledges the concern of some for-profit organizations that the concept of purely academic research may be diluted by the close ties of some not-for-profit organizations with for-profit entities, such as research sponsors and spin-off companies in which such organizations take equity. Of concern to would-be providers is the loss of control over a proprietary research tool that, once shared with a not-for-profit institution for academic research, results in commercialization gains to the providers' for-profit competitors. Funded institutions must be sensitive to this legitimate concern if for-profit organizations are expected to share tools freely.

For-profit organizations, in turn, must minimize the encumbrances they seek to impose upon not-for-profit organizations for the academic use of their tools. Reach-through royalty or product rights, unreasonable restraints on publication and academic freedom and the improper valuation of tools impede the scientific process whether imposed by a not-for-profit or for-profit provider of research tools. In fact, reach-through royalties in research tool licenses have started to attract the attention of the courts as possible examples of patent misuse and antitrust violations [15]. While these principles are directly applicable only to recipients of $\mathrm{N} \mathrm{IH}$ funding, it is hoped that other not-for-profit and for-profit organizations will adopt similar policies and refrain from seeking unreasonable restrictions or conditions when sharing materials.

Principle 4: Ensure dissemination of research resources developed with $\mathrm{NIH}$ funds Progress in science depends upon prompt access to the unique research resources that arise from biomedical research laboratories throughout government, academia, and industry. Ideally, these new resources flow to others who advance science by conducting further research. Prompt access can be accomplished in a number of ways, depending on the type of resource that has been developed, whether it has broad or specific uses, and whether it is immediately useful or needs private sector investment to realize its usefulness. The goal is the widespread, timely distribution of tools for further discovery. When research tools are used only within one or a small number of institutions, there is a great risk that fruitful avenues of research will be neglected.

Unique research resources, such as tools arising from $\mathrm{NIH}$-funded research, are to be made available to the scientific research community. Recipients are expected to manage interactions with third parties that have the potential to restrict the ability of the recipients to disseminate research tools developed with $\mathrm{NIH}$ funds. For example, a funding recipient might use the $\mathrm{N} I \mathrm{H}$ funds with funds from one or more thirdparty sponsors, or acquire a research tool from a third-party provider for use in an $\mathrm{N} I \mathrm{H}$-funded 
research project. Either situation may result in a funding recipient incurring obligations to a third party that conflict with the obligations of the funding recipients to the NIH. To avoid inconsistent obligations, funding recipients are encouraged to share these principles with potential co-sponsors of research projects and thirdparty providers of materials.

Institutions should also examine and, where appropriate, simplify the transfer of materials developed with NIH funds to for-profit institutions for internal use by those institutions. The $\mathrm{N} \mathrm{IH}$ endorses the distinguishing of internal use by for-profit institutions from the right to commercial development and sale or provision of services. In instances where the for-profit institution is seeking access for internal use purposes, funding recipients are encouraged to transfer research tools developed with $\mathrm{NIH}$ funding to such institutions without seeking option rights or royalties on the final product.

\section{Usefulness of NIH Research Tools Policy} to personalized medicine $R \& D$

These principles highlight the concerns that the free flow and access of research resources needs to be maintained for research to effectively and efficiently move forward to benefit the public health, whether for personalized medicine or other applications. Yet, certain legal encumbrances could hinder these objectives. Therefore, it is important for scientists to be cognizant of severe restrictions on the use of materials and distribution limitations for new tools and derivatives that might have a negative impact on the research enterprise. Furthermore, care needs to be exercised to retain appropriate ownership of inventions, as well as to avoid liability for overlapping agreement obligations in which a technology might be inadvertently promised exclusively to multiple parties.

It should be noted that these principles do not discourage patenting nor do they prohibit exclusive licensing; but, rather the policy encourages strategic patenting and strategic licensing. For example, exclusive licensing may be appropriate to tool companies for product development and broad dissemination to the research community. Furthermore, this policy does not support holding a technology for defensive/blocking purposes but, rather, it encourages dissemination of the technology. Tools relevant to genomics and personalized medicine research (innovations, such as laboratory techniques, bulk sequences, plasmids, cloning vectors, libraries, databases, and software) more often than not do not require patent protection. These commercially viable innovations can still be licensed without IP and continued publications on their improvements can reach many who may benefit from them. M inimizing certain unnecessary patent prosecution approaches can conserve funding for additional research efforts.

NIH practices: applying the principles Future advances in genomic research and personalized medicine will require greater partnership between government labs, universities and industry. W herever possible, it will be necessary to foster a philosophy of wide access for research purposes. For example, even in its exclusive product commercialization licenses, the $\mathrm{NIH}$ retains the right to grant additional licenses for internal research, whether such research is to be conducted at an academic or corporate facility. The NIH's philosophy of wide access for research purposes is borne out through various examples. For a decade now, the N IH has taken several actions to try to demonstrate its practices relating to the underlying principles of the Research Tools Policy [11]. The instances below have particular relevance to pharmacogenomics and personalized medicine:

- withdrawal by the NIH of its EST patent applications (1994-1995) due to issues regarding utility. The US Patent \& Trademark O ffice later issued utility guidelines for such applications corresponding to the $\mathrm{N} \mathrm{IH}$ position $[16,17,103]$

- new patent and licensing policies (1995) that limit filings on intramural NIH research tools and provide for their non-exclusive licensing without reach-through restrictions [104,105]

- UBMTA signed (1995) for standardized material exchanges among non-profit organizations $[101,106]$

- establishment of the $M$ aterials-Cooperative Research And Development Agreement (M-CRADA) that gives for-profit providers certain rights for unique materials sent to the N IH (1996) [107]

- providing NIH grantees authority to retain title, license and distribute unpatented research tools (1996) [18]

- ensuring rapid public release of data for genome sequence research grants (1996) [108]

- establishing new NIH intramural sharing policy (1997) for availability of transgenic and knockout mouse models [109] 
M ost notably, in the spirit of NIH policies, the Bayh-D oleAct was amended in 2000 to promote its utilization and commercialization goals 'without unduly encumbering future research and discovery' [19]. O ther notable projects include:

- The H GP [110]. The H GP is a significant example of the principles in action relating to the advancement of scientific knowledgeand public health due to its policy of open data release to facilitate the use of valuable sequence data by researchers around the world. Today, the data from the complete sequence of the human genome (2003) stimulate innovation in all areas of genomics, including pharmacogenomics.

- The International $\mathrm{H}$ apM ap Consortium [111]. The National Human Genome Research Institute (N H GRI) of the $\mathrm{NIH}$, which played a leading role in the HGP, is now partnered with several major international research institutions to create a haplotype map of blocks of SN Ps along the human genome. Genotypic data gathered across global ethnic populations (European, African, and East Asian) will help to correlate associations between genetic variability and diseases, or responses to drugs, vaccines and environmental agents, and be available in the public domain. As a research tool, the HapMap greatly advances the discoveries in pharmacogenomics.

- TheN ational Center for Biotechnology Information (NCBI) [112]. The NCBI is part of the $N$ ational Library of M edicine, and it houses several public genome data repositories, including the GenBank, the EST database (dbEST), the SN P database (dbSN P) and the database for human major histocompatibility complex (dbM HC).

Other practices: applying the principles outside the NIH

0 ther practices in managing research tools applicable to personalized medicine originating outside the NIH also reflect similar philosophies. Two examples include the public dbEST [113] and the SN P Consortium Ltd [114].

During the debate over EST patentability, collaboration between M erck and $\mathrm{C}$ o., Inc, Washington University and the University of Pennsylvania enabled the creation of a public EST database [113]. Early on, these partners saw the value of ESTs as tools for downstream research rather than as patentable material.

Recognizing the constraints that patents might pose to drug discovery based on pharmacogenomic markers, a group of pharmaceutical companies established the SN P Consortium (a non-profit foundation) in 1999 [114]. Collaborating with major research centers, the Consortium is geared to develop a high-quality, high-density SN P map that will be available as a research tool to the public and accessible worldwide. In this example, these for-profit entities recognized the potential of greater returns downstream from this precompetitive technology if the SN P data were made accessible as a research tool. This case shows the appropriate management of new technology by knowing when to consider an innovation as a 'tool' versus a 'product' and handling the IP accordingly.

Outlook - the future of

pharmacogenomics and the changing paradigm

The full scope of what pharmacogenomics can bring to the future of research, medicine and healthcare remains to be seen. H owever, the early signs of promise lend much optimism, so that rapid access to current and emerging research tools remains as important as ever. With the advantages of personalized medicine lying in greater safety and efficacy, the pharmaceutical industry stands to gain from reduced complications due to ADR and from the possibility of reaching nearly all patient populations by the complementary approach of creating blockbusters for the majority responder class and the customized multi-busters for the minority non-responder and adverse-responder classes [20-22]. U se of genomic, proteomic and metabolomic biomarkers that distinguish between healthy and diseased states can greatly advance the field of pharmacogenomics and bring customized medicine for the small populations of non-responders and adverse responders. This is the case with trastuzumab (H erceptin $\left.{ }^{\circledR}\right)$, a humanized monoclonal antibody from $G$ enentech approved by the FD A in 1998 for the treatment of human epidermal growth factor receptor-2 (H ER 2)-positive metastatic breast cancer (either al one or in conjunction with standard anticancer drugs like paclitaxel). D evelopment of trastuzumab as a drug was hindered by its narrow patient population (as low as $6 \%$ of the total patient population). U se of trastuzumab was advanced when it was found to bind specifically to the product of the HER2 gene selectively amplified in HER2positive patients. This is an example where 


\section{Highlights}

\section{Key conclusions}

- With the advent of pharmacogenomics, personalized medicine can become an achievable reality. Because of the increased dependency of this work upon access to research tools, scientific cooperation is needed.

- Commercial products generated as end points of one research project can be the research tools for the next step of advancement; hence, proprietary technologies need to be appropriately protected and strategically licensed to balance competitive innovation with research freedom.

- Implementation of the Bayh-Dole Act and supporting the open research enterprise can actually be complementary goals.

- Advances in public health needs research and innovation to develop freely. Such developments are becoming increasingly common in the field of pharmacogenomics through strategic partnerships between academia and industry (as cited in this article).

\section{Unresolved issues}

- Pharmacogenomics has been received both with exhilarated enthusiasm, as well as with reserved optimism, and some wonder if the benefits of personalized medicine are as great as they are touted to be; the future largely depends on how biomedical research and associated IP are appropriately utilized and judiciously managed.

- There is some concern within the pharmaceutical industry as to whether personalized medicine might destroy the future of blockbuster drugs. Another prediction is that personalized medicine will fill the often sizeable gap of non-responding patients where blockbusters fail and may even increase the marketability of existing drugs in development that would not otherwise be utilized due to adverse effects (such as in the case of trastuzumab).

\section{Points for emphasis of work in future}

- IP in the field of biomedical research and drug discovery needs to be actively managed - this includes continuing to build effective partnerships that allow sharing of research resources and competitive innovation to proceed side by side; for this, public and private enterprises need to continue to work together to develop additional strategies for furthering the research enterprise and handling associated IP appropriately.

pharmacogenomic diagnostics based on the biomarker HER2 gene product, coupled with trastuzumab holds great promise.

Some argue that such a customized approach will require less $R \& D$ investment due to smaller clinical trials and fewer regulatory hurdles. Exclusion of non-responders from large-scale clinical trials by genomic prescreening would optimize the safety and efficacy of the drug in these trials. of course, prior to that there are a number of legal, social, regulatory and ethical considerations that need to be resolved. $\mathrm{O} n$ the regulatory side, the FDA has voiced its support for the role of pharmacogenomics in clinical trials and drug development and is looking at such data, when available [23]. O $\mathrm{n}$ the other hand, because customized drugs only cater to a narrow segment of the population, others argue that the cost of bringing such drugs to the market would disinterest small pharmaceutical companies. $\mathrm{H}$ ence, they have expressed concern that costs would be higher and only few could afford personalized medicine. O riginally, pharmaceutical companies themselves were concerned that test- ing for non-responders would shrink the market size for their blockbuster drugs. H owever, combining pharmacogenomic diagnostic kits with their therapeutics are now seen as creating new market potentials.

Pharmacogenomics and personalized medicine creates greater interdependence between the biotechnology industry and the pharmaceutical industry - the former producing the upstream research tools that enable the latter to develop downstream products. This creates a dichotomy in perspectives with regard to IP [24]. For academic laboratories and small biotechnology companies, the end products of $R \& D$ are often genomic data and diagnostic genomic markers - subject inventions often worthy of patent protection for commercial development and financial gain. For the pharmaceutical companies, these same products are research tools for downstream drug development. H ence, they have greater incentive to help keep these resources open to be shared, so as to be available when needed and to manage the time and cost of $R \& D$ for the ultimate drug products. G reater 
availability also assists academic laboratories in their ongoing research. 0 verall this is a new paradigm in the drug industry with relation to IP, as seen in the case of the SN P Consortium [25]. If pharmacogenomic-based tests and associated therapeutics are sold hand-in-hand, there may be an opportunity for IP sharing between the upstream and downstream partners in drug discovery and development.

Whatever the issues, it is public health that ultimately stands to prosper from the fruits of pharmacogenomics and the research tools and resources that are a direct result there from. The patient of the 21st century will be armed with more choices and have greater access to information in individualized healthcare. For this ultimate ben efit to the society, use of the $\mathrm{NIH}$ Research Tools Policy or similar IP management strategies remains paramount in maintaining research tools and resources as readily available and reasonably accessible to the scien- tific community for furthering research on personalized medicine.

\section{Bibliography}

Papers of special note have been highlighted as either of interest $(\bullet)$ or of considerable interest $(\bullet)$ to readers.

1. Yoshida A, H uang IY, Ikawa M : M olecular abnormality of an inactive aldehyde dehydrogenase variant commonly found in O rientals. Proc. Natl. Acad. Sci. U SA 81, 258-261 (1984).

2. Garrod A: The incidence of alkaptonuria: a study in chemical individuality. Lancet 2, 1616-1620 (1902).

3. Snyder LH : Studies in human inheritance IX. The inheritance of taste deficiency in man. O hio J. Sci. 32,436-440 (1932).

4. Vogel F: [M oderne plrobleme der humangenetik] Ergeb. Inn. M ed. Kinderheilkd. 12, 52-125 (1959).

5. N ebert DW: Pharmacogenetics and pharmacogenomics: why is this relevant to clinical geneticists? C lin. G enet. 56, 247-258 (1999).

- Provides important background information, including definitions for pharmacogenetics and pharmacogenomics.

6. Laviero $M, M$ aureen $C$, Wolfgang $S$ : Pharmacogenomics: the promise of personalized medicine. AAPS PharmSci 2(1), 1-13 (2000).

-. Provides definitions and historical perspective; discusses the relevance of pharmacogenomics in drug development.

7. Pirmohamed $M$ : Pharmacogenetics and pharmacogenomics. Br. J. Clin. Pharmacol. 52, 345-347 (2001).

8. Roses A: Pharmacogenetics and the practice of medicine. $N$ ature $405,857-865$ (2000).

- Informative review on how pharmacogenomics is changing the practice of medicine.

9. $M$ ay $M$ : Pharmacogenomics lurches forward. Scientist 18(15), 26-29 (2004).
- Provides population data and the pros and cons of pharmacogenomics.

10. Bernard $\mathrm{S}$ : The five myths of pharmacogenomics. Pharmaceutical Executive 70-78 (0 ctober 2003).

- D iscusses the misconceptions associated with the technology, elaborating why it does not pose a threat to blockbuster drugs.

11. N ational Institutes of $\mathrm{H}$ ealth: Sharing biomedical research resources: principles and guidelines for recipients of $\mathrm{N} \mathrm{IH}$ research grants and contracts. US Federal Register 64(246), 72090-72096 (1999).

- This publication, directed toward recipients of N IH Research Grants and C ontracts, describes principles and guidelines for sharing biomedical resources/research tools and demonstrates the demand for balancing research needs and associated benefits with appropriate management of IP, while meeting BayhD ole obligations. It further provides examples of licensing strategies that are of importance, such as the use of the Simple Letter Agreement and the U BM TA.

12. N ational Institutes of $\mathrm{H}$ eal th: D eveloping sponsored research agreements: considerations for recipients of $\mathrm{N} \mathrm{IH}$ grants and contracts. U S Federal Register 59(215), 55674-55679 (1994).

- This publication, directed toward recipients of NIH research grants and contracts, describes important points that recipients should take into consideration when entering into sponsored research agreements with commercial entities.

13. Bayh-D ole Act: PL 96-517, Patent and Trademark Act Amendments of 1980. 35 U SC 200-212 (1980).

- This document is the key legislation that provides grantees and contractors, both for profit and not for profit, the authority to retain title to government-funded inventions, and provides them with the associated responsibilities to promote utilization, commercialization and public availability of inventions without unduly encumbering future research and development; this is pivotal legislation that changed the face of technology transfer.

14. Ferguson SM, Kim JP: Distribution and licensing of drug discovery tools - N IH perspectives. D rug $D$ iscov. Today 7(21), 1102-1106 (2002).

-. This article describes how the NIH is implementing its Research Tools Policy within its own grants and programs to allow discoveries to proceed.

15. Bayer $A G$ versus $H$ ousey $P$ harmaceuticals, Inc., 169 F. Suppl. 2d 328 (2001).

16. H offert SP: U SPTO issues Biotech Patent Guidelines. Scientist 12(14), 6 (1998).

17. Smith GK, Kettelberger D M : Patents and the H uman G enome Project, AI PLA QJ 22(1), 27-64 (1994).

18. N IH procedures for handling non-election of title to patentable biological materials. NIH Guide 25(16) (1996).

19. Transfer Commercialization Act of 2000 (Public Law 106-404).

20. M onari G-L: From blockbusters to multibusters. M edAdN ews 22(6), 1 (2003).

-D D iscusses how pharmacogenomics is filling the much needed demand for multibuster drugs that work for select populations and is helping to resurrect drugs that would otherwise have low marketability (such as trastuzumab). M ost importantly, it discusses how end products for one commercial entity could be tools for another and how such entities might partner to work synergistically.

21. Willis RC, Lesney M S: Pharmacogenomics: looking toward the payoff. M odern D rug D iscovery 30-34 (July 2003).

- D iscusses where the benefits in pharmacogenomics might be and the industrial alliances being formed to reap these benefits and to share the wealth. 
22. O estreicher $\mathrm{P}$ : Gaining market advantage through genomics. Scrip M agazine (April 2002).

23. FD A issues guidance on pharmacogenomics data. FD A M edia Release (N ovember 2003).

24. Eisenberg RS: The shifting functional balance of patents and drug regulation. $H$ ealth Aff. 20(5), 119-135 (2001).

-. Discusses the interplay of IP laws and regulatory regimes in drug development and the changing paradigm with the advent of pharmacogenomics; of particular interest is the example of research tool sharing policy established by the SN P consortium.

25. Eisenberg RS: W ill pharmacogenomics alter the role of patents in drug development? Pharmacogenomics 3(5), 571-574 (2002).

-D D escribes how pharmacogenomics and conventional methods of drug discovery may complement each other to bring diagnostics and therapeutics to the market; in particular, to capture the small population of nonresponders that previously did not benefit from blockbuster drugs.
Websites

101. http://ott.od.nih.gov/N ewPages/U BM TA.pdf The UBM TA.

102. http://ott.od.nih.gov/N ewPages/ SimplLtrAgr.pdf

The Simple Letter Agreement.

103. http://www.ama-assn.org/ama/pub/ article/2036-3603.html

Report 9 of the Council on Scientific Affairs (I-00): Patenting of $\mathrm{G}$ enes and their Mutations.

104. http://ott.od.nih.gov/N ewPages/200po6.htm PH S Patent Policy.

105. http://ott.od.nih.gov/N ewPages/300lo6.htm PH S Licensing Policy.

106. http://www.autm.net/index_ie.html Website of the Association of University Technology $M$ anagers.

107. http://ott.od.nih.gov/ $\mathrm{N}$ ewPages /M atCRAD A.pdf NIH M odel M-CRADA.

108. http://www.nhgri.nih.gov/ Website of the N H GRI, NIH .
109. http://www1.od.nih.gov/oir/sourcebook /ethic-conduct/resources.htm N IH IRP Guidelines for the Availability of Transgenic/K nockout Animals.

110. http://www.genome.gov/10001772 The homepage of the N IH N H GRI.

111. http://www.genome. gov/page. $f m$ ?pagel $D=1$ 0001688

The N IH N H GRI International H apM ap Project.

112. http://www.ncbi.nlm.nih.gov/ The homepage of the N CBI.

113. http://www.ncbi.nlm.nih.gov/Web/W hats_N ew/Announce/merck_feb10_95.html $M$ erck gene index data released to public databases: cooperative effort promises to speed scientific understanding of the human genome.

114. http://snp.cshl.org/about/ The homepage of the SN P C onsortium Ltd. 